\title{
Prevalence of food addiction and its association with anxiety, depression, and adherence to social distancing measures in Brazilian university students during the COVID-19 pandemic: a nationwide study
}

\author{
André Eduardo da Silva Júnior ${ }^{1}\left[\right.$. Mateus de Lima Macena ${ }^{2} \mathbb{D}$. Ana Debora Santos de Oliveira² ${ }^{2}$. \\ Dafiny Rodrigues Silva Praxedes ${ }^{2}\left[\right.$. Isabele Rejane de Oliveira Maranhão Pureza' ${ }^{1}$.

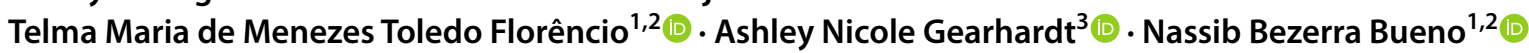

Received: 17 June 2021 / Accepted: 29 November 2021 / Published online: 8 January 2022

(C) The Author(s), under exclusive licence to Springer Nature Switzerland AG 2021

\begin{abstract}
The study's objective was to determine the prevalence of food addiction (FA) in Brazilian university students and to verify whether there is an association with anxiety, depression, and adherence to the social distancing measures adopted during the COVID-19 pandemic. This is a nationwide cross-sectional study carried out through the application of online questionnaires. Self-reported data on age, sex, economic class, race/skin color, anthropometric data, depression diagnosis, anxiety, modified Yale Food Addiction Scale 2.0 (mYFAS 2.0), and adherence to social distancing measures were collected. 5368 participants were included, with a mean age was $24.1 \pm 6.3$ years, the majority were female $(n=3990 ; 74.3 \%)$, and the mean BMI in our sample was $24.5 \pm 5.3 \mathrm{~kg} / \mathrm{m}^{2}$. The prevalence of FA was $19.1 \%$ (95\% CI: $\left.18.0 ; 20.0 \%\right)$. An association was observed between FA with depression (PR: 1.60; 95\%CI: $1.43 ; 1.78 ; p<0.01$ ), and anxiety $(3.13 ; 95 \%$ CI: $2.74 ; 3.58 ; p<0.01)$, but not with adherence to social distancing measures $(p=0.70)$. In conclusion, there was a higher prevalence of FA in Brazilian university students. Besides, university students with anxiety, depression, overweight, or obesity and females are more prone to FA. Level of evidence Level V, Opinions of authorities, based on descriptive studies, narrative reviews, clinicalexperience, or reports of expert committees.
\end{abstract}

Keywords Food addiction - Generalized anxiety disorder · SARS-CoV-2

\section{Introduction}

The World Health Organization (WHO) declared the new coronavirus disease (COVID-19) a pandemic on March 2020 due to its rapid spread among countries around the world and the important morbidity and mortality attributed to the disease [1]. In the absence of proven effective treatments, countries have adopted measures, such as the use of personal

Nassib Bezerra Bueno

nassib.bueno@fanut.ufal.br

1 Escola Paulista de Medicina, Universidade Federal de São Paulo, São Paulo, SP, Brazil

2 Faculdade de Nutrição, Universidade Federal de Alagoas, Maceió, AL 57072-900, Brazil

3 Department of Psychology, University of Michigan, Ann Arbor, MI, USA protective equipment (i.e., masks), encouraging hand washing, and measures to reduce contact between people, such as quarantine, social distancing, and social isolation [2, 3]. Schools, universities, gyms, restaurants, and commercial activities had their activities suspended or transferred to a remote system promoting changes in the lifestyle of individuals [4].

Although extremely necessary, the public health measures adopted to contain the spread of SARS-CoV-2 and its consequences impacted the mental health of individuals, reflected in more significant psychological distress, increased anxiety, and depressive symptoms in the population [5]. High rates of anxiety and depression were observed in several countries among university students, leading to concerns with their health and their families, such as difficulties in concentration, decreased social interactions, poor academic performance, decreased family income, and future employment 
[6-9]. The scenario composed of psychological stress, increased levels of anxiety and depression, and decreased health care, is conducive to the emergence or relapse of disordered eating behaviors and addictions [10, 11].

The body of evidence regarding the food addiction construct has been growing in recent years. Food addiction is characterized by excessive consumption of energetically dense, hyper-palatable, and processed foods with characteristics and repercussions similar to substance addiction [12]. Given the complex nature of addictions, food addiction is assessed with instruments designed to reflect this diagnostic criterion for other addictive disorders, such as loss of control over consumption, intense cravings, and continued use despite negative health consequences [13]. In 2009, the Yale Food Addiction Scale (YFAS) was the first psychometrically sound tool designed to specifically assess food addiction by applying the diagnostic criteria for substance dependence to the consumption of hyper-palatable foods (e.g., chocolate, pizza) [14]. The mean prevalence of food addiction in the general population is $19.6 \%$, and that this diagnosis is more frequent in women (24.9\%), individuals over the age of 35 years (22.2\%), with overweight/obesity (24.9\%), and in individuals diagnosed with eating disorders (57.6\%) [13]. In Brazil, two studies evaluated food addiction in adults before the COVID-19 pandemic. The first observed a prevalence of $4.3 \%$, and the second, carried out with a group of medical students, a prevalence of food addiction was $8.6 \%[15,16]$.

Food addiction seems to be involved in the genesis of obesity [17]. In a systematic review that included 25 studies aimed to assess food addiction and its relationship with other variables, food addiction was associated with a higher body mass index (BMI) [13]. In addition, food addiction was also associated with anxiety, depression, and binge eating [18]. Faced with the closure of universities, the current pandemic is increasing depression and anxiety levels in university students [19], which may increase the likelihood of experiencing symptoms of food addiction. Understanding the prevalence of food addiction in undergraduate students may guide the need for policies and treatment to reduce addictive eating in this group. Hence, the objectives of the present study were to determine the prevalence of food addiction in Brazilian university students during the COVID-19 pandemic and verify whether there is an association between the prevalence of food addiction with anxiety, depression, and adherence to the social distancing measures adopted during the COVID-19 pandemic.

\section{Methods}

\section{Ethical aspects}

This study was approved by the Research Ethical Committee of the Universidade Federal de Alagoas (protocol number: 4.410.403). All participants were presented with the online consent form on the first page of the online questionnaire, and it was necessary to accept it to access the questionnaire.

\section{Study design}

Cross-sectional study.

\section{Location and sample}

The Federative Republic of Brazil is the largest country in Latin America, with continental dimensions. Administratively, the country is divided into 27 federative units that make up the five macro-regions of Brazil: North, Northeast, Midwest, Southeast, and South.

Students were recruited from 94 universities in the Brazilian public and private education network, distributed among all federative units. Students of both sexes and aged between 18 and 59 years were included. Individuals who underwent bariatric surgery and pregnant or lactating women were not included. Individuals who did not complete the questionnaire and those who reported improbable values for the variables were excluded.

\section{Sample size}

For the sample size calculation, $95 \%$ confidence and $1 \%$ acceptable margin of error were adopted. A population size of 8,449,521 individuals, corresponding to the number of students enrolled in universities in Brazil in 2018, was considered [20]. The expected frequency of food addiction was $10 \%$. This value was based on the expectation that the measures of social distancing, and the consequent impact on depression and anxiety in the population, would show a higher prevalence than that observed in a study carried out in Brazil that evaluated 7639 individuals and observed a prevalence of food addiction of $4.32 \%$ [15]. With these parameters, 3456 university students were needed to compose the sample. Given the important heterogeneity in the geographic distribution of population across the country, the sample was weighted by the number of university students in each of the macro-regions. Thus, 283 ( $8.2 \%$ of the sample) individuals were needed in the North, 736 (21.3\%) Northeast, 317 (9.2\%) Midwest, 1,536 (44.4\%) Southeast, and 584 (16.8\%) in the South. To guarantee the intra-regional homogeneity 
of the sample, a maximum ratio of 10 was assumed between the collected and calculated sample by each Federative Unit.

\section{Recruitment and procedures}

The online questionnaire for data collection was developed and tested in a pilot study to assess possible difficulties in accessing the form, understanding the questions, and estimating the time of data collection. The pilot study was conducted with 50 participants who met the inclusion criteria for this study and were linked to one of the participating institutions. These individuals were included in the final database since there were no reported difficulties with the assessed questionnaire. The average response time of the questionnaire in the pilot study was $9 \mathrm{~min}$ and $24 \mathrm{~s}$.

Data collection took place by completing an online questionnaire between October 27 and December 11 (between epidemiological weeks 44 and 50 of 2020). Brazil presented its first case and the first death of COVID-19 in the 10th and 12th epidemiological week of 2020, respectively. During 2020 , the country reached the first peaks of new cases and deaths from COVID-19 in the 30th epidemiological week, with absolute numbers of 319,653 cases and 7677 deaths. After a decline in these numbers, Brazil experienced a second wave of the disease with 333,028 new cases and 5233 deaths due to COVID-19 in the 51st epidemiological week of 2020 [21].

The recruitment of participants took place through invitations sent by e-mails to university leaders, to the students, institutional websites, and social media. Thus, the participants knew the research topic (food addiction in Brazilian university students) and the research objectives.

\section{Demographic and clinical variables}

All participants were asked to report their age (in years), date of birth, sex, the educational institution they were affiliated with, the federative unit, and race/color [white (Caucasian), black (Afro-descendant), brown, yellow (Asian), indigenous]. Furthermore, the participants self-reported whether they had received a medical diagnosis of depression.

\section{Economic class}

The Critério de Classificação Econômica Brasil was used to determine the economic class of the individuals. This instrument consists of questions about ownership of assets, the number of bathrooms in the home, the level of education of the head of the family, and access to public services such as running water. The sum of the scores is made for each individual, which can vary from 0 to 100 points (the higher the score, the higher the estimated average household income), and were classified into six economic classes: "A" (45-100 points), "B1" (38-44), "B2" (29-37), “C1” (23-28), "C2" (17-22), "D-E" (0-16) [22].

\section{Anthropometry}

Height (in meters) and body weight (in kilograms) data were self-reported during the online questionnaire. The Body Mass Index (BMI) was calculated and classified as underweight (BMI $<18.5 \mathrm{~kg} / \mathrm{m}^{2}$ ), normal weight (BMI between 18.5 and $24.9 \mathrm{~kg} / \mathrm{m}^{2}$ ), overweight (BMI between 25.0 and $\left.29.9 \mathrm{~kg} / \mathrm{m}^{2}\right)$, and obesity (BMI $\left.\geq 30 \mathrm{~kg} / \mathrm{m}^{2}\right)$.

\section{Adherence to social distancing measures}

Adherence to social distancing measures was also selfreported and collected through the question "Did you follow social distancing measures?", Participants could answer "I followed social distancing most of the time, only going to supermarkets and pharmacies", "I followed the social distancing, but I had to leave to work" or "I did not follow the social distancing".

\section{Signs and symptoms of anxiety disorder}

The Generalized Anxiety Disorder 7-item scale (GAD-7) was developed to diagnose generalized anxiety disorder [23]. This tool has a record of evidence of validity in Brazil [24]. The scale consists of seven items, arranged in four points from 0 to 3, where 0 represents "no time" and 3 "almost every day", the score is added and can vary from 0 to 21 points, is considered an indicator of positive signs and symptoms of anxiety disorder, scores equal to or greater than 10 [23]. Cronbach's $\alpha$ for this sample was 0.89 .

\section{Food addiction}

Food addiction was measured using the modified Yale Food Addiction Scale 2.0 (mYFAS 2.0) [25], which is cross-culturally validated for the Portuguese language [26]. It consists of 13 questions, of which 11 represent symptoms related to the individual's eating behavior related to aspects of disorders related to substance use in the DSM-V, and 2 refer to the clinical impairment/distress. Each question is answered according to the frequency at which it occurs, ranging from "never" to "every day", and there is a threshold proposed for each of them so that the criteria for the symptom can be met. Cronbach's $\alpha$ for this sample was 0.88 .

In the end, the 11 symptoms are added to create a symptom count scoring option. Individuals who have a symptom count of two or more and meet the threshold for either of the clinical impairment/distress questions are classified as having a food addiction. Also, it is possible to classify it as without food addiction or with mild, moderate, or severe 
food addiction (mild $=2-3$ symptoms plus impairment or distress, moderate $=4-5$ symptoms plus impairment or distress, severe $=6$ or more symptoms plus impairment or distress) [25].

\section{Statistical analysis}

After the collection period, we verified and removed from the database duplicate or incomplete questionnaires and unlikely data. Descriptive statistics for the sample were computed and summarized in means (standard deviation, SD) for continuous variables and percentages for categori$\mathrm{cal}$ variables. Poisson regression with robust estimation of the variances was used to identify the factors associated with food addiction (dependent variable; "0" without food addiction and " 1 " with food addiction) in the multivariable model. As our outcome is binary, the robust estimation of the variances was necessary to overcome the fact that binary variables present a mean that is higher than the variance, violating an assumption of the Poisson model, which is that the mean and the variance of the outcome must be equal. Furthermore, we looked for failed convergence of the models.

We designed a directed acyclic graph (DAG) to illustrate the causal paths between adherence to social distancing measures and food addiction (Fig. 1). The DAG was developed with the aid of the DAGitty software [27] and aimed to guide the selection of variables included in the DAG-oriented multivariable model. Thus, the minimum set of variables to estimate the direct effect of social distancing on food addiction included age, sex, BMI classification, anxiety disorder, and depression.
For all analyses, it was adopted an alpha value equal to $5 \%$. All analyses were conducted with the aid of the statistical program R v. 3.6.1 (R Foundation for Statistical Computing, Vienna, Austria) using the packages "ggplot" to build the graph, "rcmdr" to conduct descriptive analysis, and "glm" and "sandwich" to conduct the Poisson regression analysis."

\section{Results}

6532 questionnaires were answered and returned on the online platform. Two federative units received more individuals than the previously established ratio between the collected and calculated sample of 10 . Therefore, a random draw was carried out among the participants of these two federative units, resulting in the exclusion of 586 individuals (554 from Piauí and 32 from Roraima). Figure 2 shows the flowchart of the participants.

For this analysis, 5368 participants were included, it was possible to reach the weighted sample in all country regions. Figure 3 shows the estimated population of university students by region and the percentage distribution of the sample in this research.

The average age was $24.1 \pm 6.3$ years, with the majority of the participants being female $(n=3990 ; 74.3 \%)$ and 2075 (38.7\%) from the Northeast. Most participants declared themselves to be white $(n=2766 ; 51.5 \%)$ and were from economic classes B2 and C1, with 1626 (30.3\%) and 1111 (20.7\%) individuals, respectively. The characteristics of the sample are described in Table 1. The mean BMI in our sample was $24.5 \pm 5.3 \mathrm{~kg} / \mathrm{m}^{2}$. The prevalence of food addiction
Fig. 1 Directed acyclic graph showing paths between adherence to social distancing (exposure variable; circle with an arrow) and food addiction (outcome variable; circle with the bar). Circles indicate the variables assessed in the study, and arrows indicate the causal relationship among these variables. Variable with an arrow pointing both to the exposure variable and to the outcome variable are considered a confounder and must be adjusted in the multivariable model

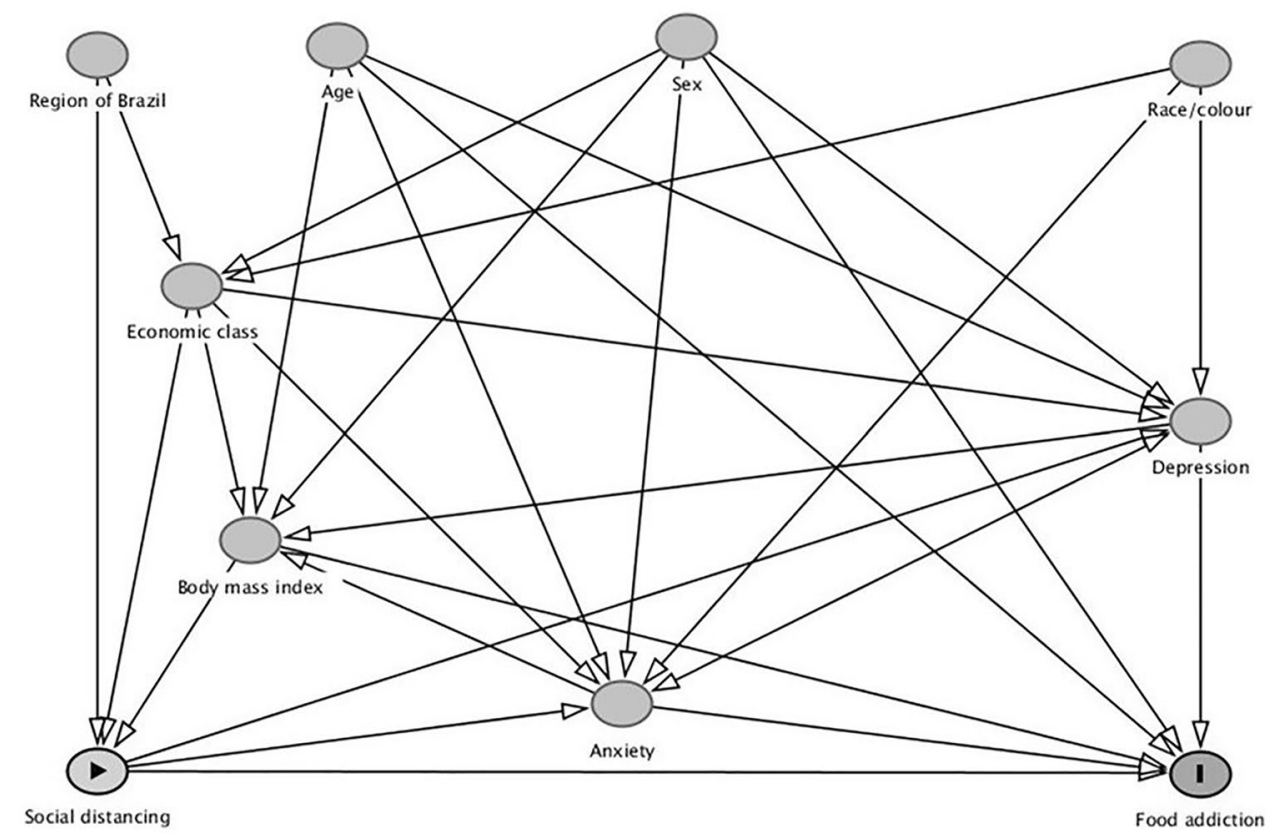


Fig. 2 Flowchart of the participants selection

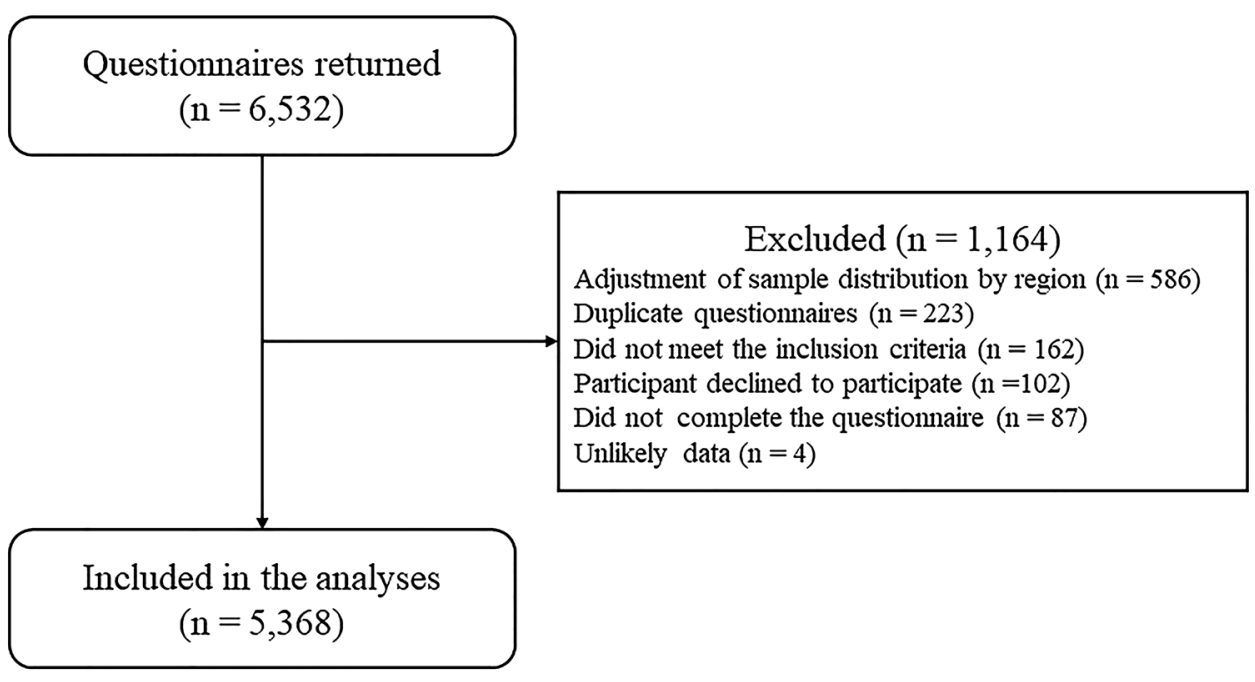

Fig. 3 The estimated university student population and sample distribution by geographic region of Brazil

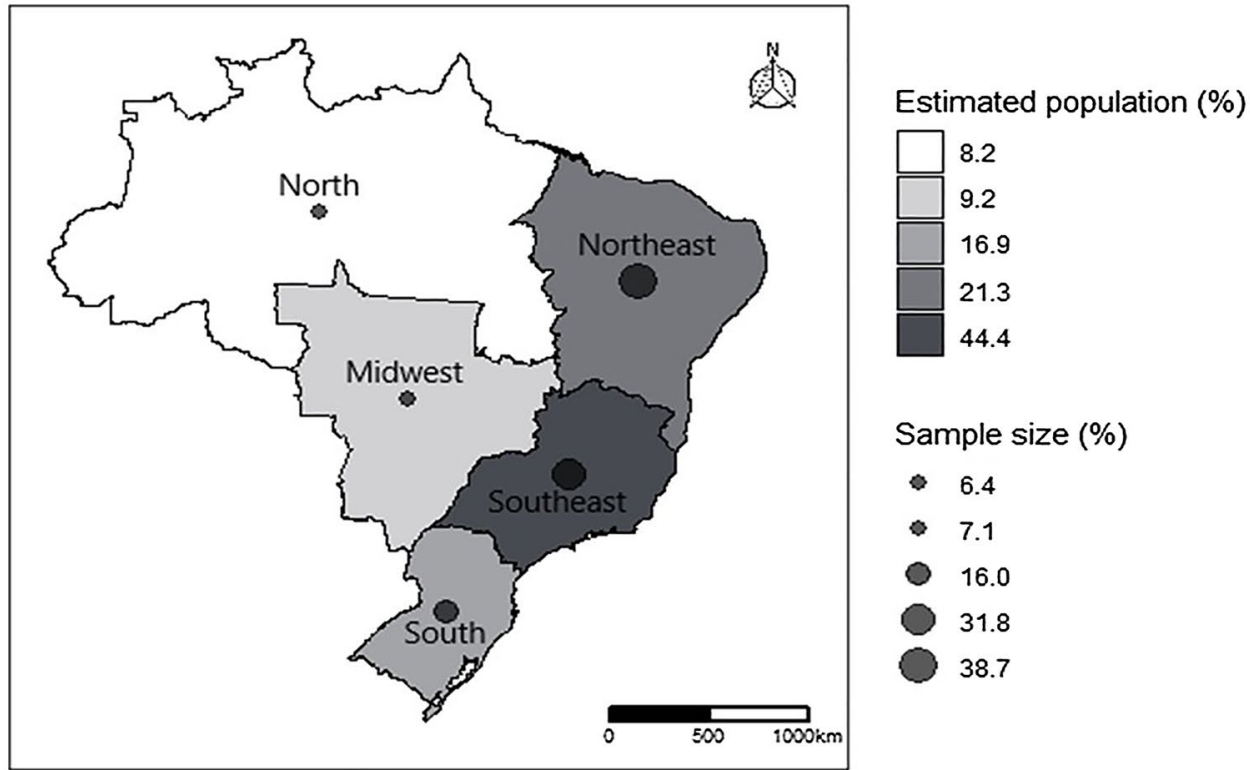

was $19.1 \%$ (95\% CI: $18.0 ; 20.0 \%$ ) and the mean mYFAS 2.0 symptom count was $1.88 \pm 2.64$ points. Regarding sex, the prevalence of food addiction in women was $21.6 \%$ (95\% CI: $20.0 ; 23.0 \%$ ) and $11.8 \%$ (95\% CI: $10.0 ; 14.0 \%$ ) in males.

The DAG-oriented multivariable analysis can be seen in Table 2. In the DAG-oriented multivariable analysis, the prevalence of food addiction remained associated with age (in years) (PR: 0.96; 95\% CI: 0.96; 0.97; $p<0.01$ ) and with females (PR: $1.54 ; 95 \%$ CI: $1.33 ; 1.78 ; p<0.01$ ), with male sex being the reference. An association was observed between food addiction and BMI categories (omnibus test: $p<0.01$ ), an association with underweight was observed (PR: $0.49 ; 95 \%$ CI: $0.33 ; 0.72 ; p<0.01$ ), overweight (PR: 2.08; 95\% CI: $1.83 ; 2.36 ; p<0.01)$ and obesity (PR: 3.17 ; $95 \%$ CI: $2.80 ; 3.59 ; p<0.01$ ), using normal weight as a reference. There was also an association between food addiction and the medical diagnosis of depression (PR: 1.60; 95\% CI: $1.43 ; 1.78 ; p<0.01$ ) and anxiety disorder (PR: $3.13 ; 95 \%$ CI: $2.74 ; 3.58 ; p<0.01)$, both with the reference category being the non-diagnostic. However, there was no association between food addiction and adherence to social distancing measures (omnibus test: $p=0.70$ ). Non-adherence (PR: 1.05; 95\% CI: $0.77 ; 1.42 ; p=0.73$ ), and partial adherence (i.e., leaving home to work) to social distancing (PR: 1.04; 95\% CI: $0.93 ; 1.17 ; p=0.42$ ), did not show different prevalence when compared to total adherence (i.e., leaving home only for going to the supermarket and pharmacy). 
Table 1 Sample characteristics according to demographic, social, economic, clinic and anthropometric variables

\begin{tabular}{|c|c|c|}
\hline \multirow[t]{2}{*}{ Variables } & \multicolumn{2}{|c|}{$\begin{array}{l}\text { Sample } \\
(n=5368)\end{array}$} \\
\hline & $N$ & $\%$ \\
\hline \multicolumn{3}{|l|}{ Sex } \\
\hline Male & 1378 & 25.7 \\
\hline Female & 3990 & 74.3 \\
\hline \multicolumn{3}{|l|}{ Region } \\
\hline North & 346 & 6.4 \\
\hline Northeast & 2075 & 38.7 \\
\hline Midwest & 379 & 7.1 \\
\hline Southeast & 1707 & 31.8 \\
\hline South & 861 & 16.0 \\
\hline \multicolumn{3}{|l|}{ Race/skin color } \\
\hline White (Caucasian) & 2766 & 51.5 \\
\hline Black (Afro-descendant) & 658 & 12.3 \\
\hline Brown & 1852 & 34.5 \\
\hline Yellow (Asian) & 64 & 1.2 \\
\hline Indigenous & 28 & 0.5 \\
\hline \multicolumn{3}{|l|}{ Economic class } \\
\hline A & 763 & 14.2 \\
\hline B1 & 876 & 16.3 \\
\hline B2 & 1626 & 30.3 \\
\hline $\mathrm{C} 1$ & 1111 & 20.7 \\
\hline $\mathrm{C} 2$ & 719 & 13.4 \\
\hline D-E & 273 & 5.1 \\
\hline \multicolumn{3}{|l|}{ Body mass index } \\
\hline Underweight & 401 & 7.5 \\
\hline Normal weight & 2988 & 55.7 \\
\hline Overweight & 1210 & 22.5 \\
\hline Obesity & 769 & 14.3 \\
\hline \multicolumn{3}{|l|}{ Diagnosis of depression } \\
\hline No & 4579 & 85.3 \\
\hline Yes & 789 & 14.7 \\
\hline \multicolumn{3}{|l|}{ Anxiety disorder } \\
\hline No & 3058 & 57.0 \\
\hline Yes & 2310 & 43.0 \\
\hline \multicolumn{3}{|l|}{ Food Addiction } \\
\hline Non-food addiction & 4344 & 81.0 \\
\hline Mild food addiction & 222 & 4.1 \\
\hline Moderate food addiction & 249 & 4.6 \\
\hline Severe food addiction & 553 & 10.3 \\
\hline \multicolumn{3}{|l|}{ Social distancing } \\
\hline $\begin{array}{l}\text { Adhered the social distancing for most of the time, } \\
\text { performing only visits to supermarkets and pharma- } \\
\text { cies }\end{array}$ & 3947 & 73.6 \\
\hline $\begin{array}{l}\text { Adhered the social distancing, but had to leave to } \\
\text { work }\end{array}$ & 1253 & 23.3 \\
\hline Did not adhere to social distancing & 168 & 3.1 \\
\hline
\end{tabular}

\section{Discussion}

To our knowledge, this is the first study that assessed the prevalence of food addiction during the COVID-19 pandemic and its relationship with anxiety, depression, and adherence to social distancing measures in university students. In the present study, the prevalence of food addiction was $19.1 \%$ (95\% CI: 18.0; 20.0\%). Besides, food addiction was not associated with adherence to social distancing measures, even after adjusting in DAG-oriented multivariable analysis for age (in years), sex, BMI, diagnosis of depression, anxiety. However, our findings indicate that during the COVID-19 pandemic, university students with anxiety, depression, overweight, or obesity and females are more prone to food addiction.

The prevalence of food addiction observed in this study is higher than that found in other studies carried out in Brazil before the new coronavirus pandemic. Cardoso et al. [16] in a study conducted with 150 Brazilian medical students, with an average age of $22.7 \pm 3.7$ years, the prevalence of food addiction determined by mYFAS 2.0 was 8.6\%. Also, in Brazil, Nunes-Neto et al. [15], conducted an online study to determine the prevalence of food addiction in a large non-clinical sample, where 7639 individuals ( $71.3 \%$ females) with a mean age of $27.2 \pm 7.9$ years were assessed and found a prevalence of food addiction determined by the mYFAS 2.0 of $4.3 \%$, a value much lower than that observed in our study. It is noteworthy that we cannot attribute the higher prevalence observed in our study to the pandemic of COVID-19 since we do not have data on food addiction in a similar sample to our study before the pandemic.

The association between food addiction, anxiety (PR: 3.13 ; 95\% CI: $2.74 ; 3.58 ; p<0.01$ ) and depression (PR: 1.60 ; $95 \%$ CI: $1.43 ; 1.78 ; p<0.01)$ corroborated with the findings from a systematic review with meta-analysis, which aimed to determine the relationship between food addiction and mental health symptoms [18]. These findings added to the nonassociation with adherence to social distancing measures (omnibus test: $p=0.70$ ) point out that the most important factors for the diagnosis of food addiction in this scenario are anxiety disorder and the diagnosis of depression. Possible explanations for the discrepancy in the prevalence of food addiction between the two studies conducted before the pandemic $[15,16]$ and our study involve the impacts of social distancing measures on eating behavior. Changes in lifestyle, study, and work routines and reduced social contact imposed by measures to contain the spread of SARS-CoV-2 can lead to substantial increases in stress and anxiety levels, well-known risk factors for eating disorders, and food addiction $[18,28,29]$. 
Table 2 DAG-oriented multivariable prevalence ratios for the food addiction in Brazilian university students according to demographic, clinics, anthropometrics, social and economic variables

\begin{tabular}{llll}
\hline Variables & \multicolumn{2}{l}{ DAG-oriented multivariable analysis ${ }^{\mathrm{a}}$} \\
\cline { 2 - 4 } & PR & $95 \%$ CI & $p$ \\
\hline Age (in years) & 0.96 & $0.96 ; 0.97$ & $<0.01$ \\
Sex & & & - \\
Male & 1.00 & - & $<0.01$ \\
Female & 1.54 & $1.33 ; 1.78$ & $<0.01$ \\
Body mass index & & & - \\
Normal weight & 1.00 & - & $<0.01$ \\
Underweight & 0.49 & $0.33 ; 0.72$ & $<0.01$ \\
Overweight & 2.08 & $1.83 ; 2.36$ & $<0.01$ \\
Obesity & 3.17 & $2.80 ; 3.59$ & - \\
Diagnosis of depression & & & $<0.01$ \\
No & 1.00 & - & - \\
Yes & 1.60 & $1.43 ; 1.78$ & $<0.01$ \\
Anxiety disorder & & & 0.70 \\
No & 1.00 & - & - \\
Yes & 3.13 & $2.74 ; 3.58$ & \\
Social distancing & & & 0.42 \\
Adhered the social distancing for most of the time, performing & 1.00 & - & \\
$\quad$ only visits to supermarkets and pharmacies & & & \\
Adhered the social distancing, but had to leave to work & 1.04 & $0.93 ; 1.17$ & $0.77 ; 1.42$ \\
Did not adhere to social distancing & 1.05 & & \\
\hline
\end{tabular}

$P R$ prevalence ratio by Poisson Regression with robust adjustment of variance; $95 \%$ CI 95\% confidence interval

${ }^{\text {a}}$ Prevalence ratios for the food addiction adjusted by age, sex, body mass index class, diagnosis of depression, anxiety disorder and adherence to social distancing measures
The COVID-19 pandemic has had significant consequences for the mental health of the population. Recently, research has shown the negative impact on the mental health of university students [6-9]. Addictions, such as excessive alcohol consumption and ingestion of hyper-palatable foods, are known as mechanisms to cope with negative emotions and stress, especially after situations, such as natural disasters, financial crises, and terrorist attacks [30-33]. The repetition of the pattern of ingestion of hyper-palatable foods may favor the development of food addiction, which can be explained by the neurobehavioral adaptations promoted by the reduction of the activity of the hypothalamic-pituitary-adrenal axis and, consequent decrease in the production of cortisol promoted by the reward associated with this food profile $[34,35]$.

As for the study's strengths, we highlight that we reached a large and representative sample of Brazilian university students across all country regions and from public and private higher education. Hence, we believe that we managed to capture the economic and ethnic diversity of Brazilian university students in our sample. Finally, our study adds novel scientific evidence about food addiction, given that to the extent of our knowledge, there are no other studies that aimed to determine the prevalence of food addiction in university students during the COVID-19 pandemic, as well as its association with anxiety, depression, and adherence to social distancing measures.

The present study has limitations. Initially, the anthropometric data were self-reported and may not precisely reflect the individual's real data. However, using of selfreported measures of body weight and height in epidemiological studies can be an alternative, considering that high concordances between self-reported and measured values have already been observed [36]. Second, the self-report of non-adherence to social distancing measures may have been influenced by moral judgment, which could induce the participant to respond that does not correspond to reality. Although, it is believed that the absence of the researcher face to face with the participant can reduce the fear of the moral judgment of non-adherence to the social distancing measures. It is highlighted that the non-use of an objective tool for the diagnosing of depression may lead to inaccurate estimates of the prevalence and possible relationships of this variable in our analyses. Another limitation refers is that the invitation sent to the participants presented the objective of the research, which was to evaluate food addiction in university students. This fact may have induced the participation of individuals who had some identification with 
the theme. Finally, considering that the prevalence of food addiction is known to be higher among women [13] and that our sample was mainly composed of females, our estimate of the prevalence of food addiction in the general sample may have been inflated.

In conclusion, there was a higher prevalence of food addiction in the present study than that found in other studies in Brazil carried out in periods before the COVID-19 pandemic. Our findings indicate that, during the COVID19 pandemic, university students with anxiety, depression, overweight, or obesity and females are more prone to food addiction. Furthermore, adherence to social distancing measures was not associated with food addiction diagnosis in university students. The current evidence about the association of anxiety and depression and the increase in the prevalence of food addiction must be considered when treating individuals with overweight and obesity in the postpandemic moment.

\section{What is already known on this subject?}

Food addiction has attracted the attention of the scientific and clinical community in recent years, and the body of evidence about its association with obesity and mental health has grown in recent years.

\section{What does this study add?}

This study shows a high prevalence of food addiction in Brazilian university students. Besides, during the COVID19 pandemic, university students with anxiety, depression, overweight or obesity, and women are more prone to food addiction. There was no association between food addiction and adherence to social distancing measures.

Acknowledgements The authors would like to thank Prof. Dr. Gabriel Soares Bádue from the Faculdade de Nutrição of the Universidade Federal de Alagoas for preparing the map.

Author contributions A.E.S.J. and M.L.M. contributed to the conception and design of the study, data collection, statistical analysis, data interpretation, and writing of the manuscript. A.D.S.O., D.R.S.P., and I.R.O.M.P. contributed to the data collection and writing of the manuscript. T.M.M.T.F., A.N.G., and N.B.B. contributed to the data interpretation, the writing of the manuscript, and critical review of the intellectual content. All authors reviewed and approved the final version of the work.

Funding A.E.S.J. is supported by the Coordenação de Aperfeiçoamento de Pessoal de Nível Superior-Brazil (CAPES) research fellowships (grant number: 88887.480702/2020-00). M.L.M. is supported by the Coordenação de Aperfeiçoamento de Pessoal de Nível Superior-Brazil (CAPES) research fellowships (grant number: 23065.005919/2021-75).
D.R.S.P. is supported by the Coordenação de Aperfeiçoamento de Pessoal de Nível Superior-Brazil (CAPES) research fellowships (grant number: $23065.005919 / 2021-75)$. I.R.O.M.P. is supported by the Coordenação de Aperfeiçoamento de Pessoal de Nível Superior-Brazil (CAPES) research fellowships (grant number: 88887.465281/2019-00).

Availability of data and material Data can be made available upon request.

\section{Declarations}

Conflict of interest The authors declare that they have no conflicts of interest.

Ethical approval The study protocol was reviewed and approved by the Research Ethical Committee of the Universidade Federal de Alagoas (process number: 4.410.403).

Informed consent Informed consent was obtained from all individual participants included in the study.

\section{References}

1. WHO. Coronavirus Disease (COVID-19)-Events as they happen. https://www.who.int/emergencies/diseases/novel-coronavirus2019/events-as-they-happen. Accessed Apr 2021

2. Feng S, Shen C, Xia N et al (2020) Rational use of face masks in the COVID-19 pandemic. Lancet Respir Med 8(5):434-436. https://doi.org/10.1016/S2213-2600(20)30134-X

3. Lewnard JA, Lo NC (2020) Scientific and ethical basis for socialdistancing interventions against COVID-19. Lancet Infect Dis 20(6):631-633. https://doi.org/10.1016/S1473-3099(20)30190-0

4. Cecchetto $C$, Aiello M, Gentili $C$ et al (2021) Increased emotional eating during COVID-19 associated with lockdown, psychological and social distress. Appetite 160:105122. https://doi.org/10. 1016/j.appet.2021.105122

5. Salari N, Hosseinian-Far A, Jalali R et al (2020) Prevalence of stress, anxiety, depression among the general population during the COVID-19 pandemic: a systematic review and metaanalysis. Global Health 16(1):57. https://doi.org/10.1186/ s12992-020-00589-w

6. Fernández RS, Crivelli L, Guimet NM et al (2020) Psychological distress associated with COVID-19 quarantine: latent profile analysis, outcome prediction and mediation analysis. J Affect Disord 277:75-84. https://doi.org/10.1016/j.jad.2020.07.133

7. Son C, Hegde S, Smith A et al (2020) Effects of COVID-19 on college students' mental health in the United States: interview survey study. J Med Internet Res 22(9):e21279. https://doi.org/ $10.2196 / 21279$

8. Sankhi S, Marasine NR (2020) Impact of COVID-19 pandemic on mental health of the general population, students, and health care workers. Europasian J Med Sci. https://doi.org/10.46405/ejms. v2i2.131

9. Cao W, Fang Z, Hou G et al (2020) The psychological impact of the COVID-19 epidemic on college students in China. Psychiatry Res 287:112934. https://doi.org/10.1016/j.psychres.2020.112934

10. Lippi G, Henry BM, Bovo C et al (2020) Health risks and potential remedies during prolonged lockdowns for coronavirus disease 2019 (COVID-19). Diagnosis 7(2):85-90. https://doi.org/10.1515/ dx-2020-0041 
11. Volkow ND (2020) Collision of the COVID-19 and addiction epidemics. Ann Intern Med 173(1):61-62. https://doi.org/10.7326/ M20-1212

12. Gearhardt AN, Davis C, Kuschner R et al (2011) The addiction potential of hyperpalatable foods. Curr Drug Abuse Rev 4(3):140 145. https://doi.org/10.2174/1874473711104030140

13. Pursey KM, Stanwell P, Gearhardt AN et al (2014) The prevalence of food addiction as assessed by the Yale Food Addiction Scale: a systematic review. Nutrients 6(10):4552-4590. https://doi.org/ 10.3390/nu6104552

14. Gearhardt AN, Corbin WR, Brownell KD (2009) Preliminary validation of the Yale Food Addiction Scale. Appetite 52(2):430-436. https://doi.org/10.1016/j.appet.2008.12.003

15. Nunes-Neto PR, Köhler CA, Schuch FB et al (2018) Food addiction: Prevalence, psychopathological correlates and associations with quality of life in a large sample. J Psychiatr Res 96:145-152. https://doi.org/10.1016/j.jpsychires.2017.10.003

16. Cardoso TQ, Pereira CW, Souza Costa T et al (2020) Translation and validation of the addiction-like Eating Behavior Scale from English to Portuguese in Brazil. J Addict Dis 38(2):113-121. https://doi.org/10.1080/10550887.2020.1724607

17. Constant A, Moirand R, Thibault R et al (2020) Meeting of minds around food addiction: insights from addiction medicine, nutrition, psychology, and neurosciences. Nutrients 12(11):3564. https://doi.org/10.3390/nu12113564

18. Burrows T, Kay-Lambkin F, Pursey K et al (2018) Food addiction and associations with mental health symptoms: a systematic review with meta-analysis. J Hum Nutr Diet 31(4):544-572. https://doi.org/10.1111/jhn.12532

19. Bruffaerts R, Mortier P, Kiekens G et al (2018) Mental health problems in college freshmen: Prevalence and academic functioning. J Affec disord 225:97-103. https://doi.org/10.1016/j.jad.2017. 07.044

20. INPE (2019) Sinopse Estatística da Educação Superior 2018. INPE, Brasília

21. Brasil. Ministério da Saúde. Painel de casos de doença pelo coronavírus 2019 (COVID-19) no Brasil (2021). https://covid.saude. gov.br. Accessed 14 Mar 2021

22. Associação Brasileira de Empresas de Pesquisas-ABEP (2020) Critérios de classificação econômica Brasil. ABEP, São Paulo

23. Spitzer RL, Kroenke K, Williams JB et al (2006) A brief measure for assessing generalized anxiety disorder: the GAD-7. Arch Intern Med 166(10):1092-1097. https://doi.org/10.1001/archinte. 166.10.1092

24. Sousa TV, Viveiros V, Chai MV et al (2015) Reliability and validity of the Portuguese version of the Generalized Anxiety Disorder (GAD-7) scale. Health Qual Life Outcomes 13:50. https://doi.org/ 10.1186/s12955-015-0244-2

25. Schulte EM, Gearhardt AN (2017) Development of the modified yale food addiction scale version 2.0. Eur Eat Disord Rev 25(4):302-308. https://doi.org/10.1002/erv.2515
26. Nunes-Neto PR, Köhler CA, Schuch FB et al (2018) Psychometric properties of the modified Yale Food Addiction Scale 2.0 in a large Brazilian sample. Braz J Psychiatry 40(4):444-448. https:// doi.org/10.1590/1516-4446-2017-2432

27. Textor J, Zander B, Gilthorpe MS et al (2016) Robust causal inference using directed acyclic graphs: the R package "dagitty." Int J Epidemiol 45(6):1887-1894. https://doi.org/10.1093/ije/dyw341

28. Brooks SK, Webster RK, Smith LE et al (2020) The psychological impact of quarantine and how to reduce it: rapid review of the evidence. Lancet 395:912-920. https://doi.org/10.1016/S01406736(20)30460-8

29. Puccio F, Fuller-Tyszkiewicz M, Ong D et al (2016) A systematic review and meta-analysis on the longitudinal relationship between eating pathology and depression. Int J Eat Disord 49(5):439-454. https://doi.org/10.1002/eat.22506

30. Keyes KM, Hatzenbuehler ML, Hasin DS (2011) Stressful life experiences, alcohol consumption, and alcohol use disorders: the epidemiologic evidence for four main types of stressors. Psychopharmacology 218(1):1-17. https://doi.org/10.1007/ s00213-011-2236-1

31. Pool E, Delplanque $S$, Coppin $G$ et al (2015) Is comfort food really comforting? Mechanisms underlying stress-induced eating. Food Res Int 76(2):207-215. https://doi.org/10.1016/j.foodres.2014.12. 034

32. Goeij MC, Suhrcke M, Toffolutti V et al (2015) How economic crises affect alcohol consumption and alcohol-related health problems: a realist systematic review. Soc Sci Med 131:131-146. https://doi.org/10.1016/j.socscimed.2015.02.025

33. Kemp E, Kennett-Hensel PA, Williams KH (2014) The calm before the storm: examining emotion regulation consumption in the face of an impending disaster. Psychol Mark 31(11):933-945. https://doi.org/10.1002/mar.20744

34. Ulrich-Lai YM, Fulton S, Wilson M et al (2015) Stress exposure, food intake and emotional state. Stress 18(4):381-399. https://doi. org/10.3109/10253890.2015.1062981

35. Yau YH, Potenza MN (2013) Stress and eating behaviors. Minerva Endocrinol 38(3):255-267

36. Davies A, Wellard-Cole L, Rangan A et al (2020) Validity of self-reported weight and height for BMI classification: a crosssectional study among young adults. Nutrition 71:110622. https:// doi.org/10.1016/j.nut.2019.110622

Publisher's Note Springer Nature remains neutral with regard to jurisdictional claims in published maps and institutional affiliations. 